To the Editor of the Mathematical Gazette

DeAR SIR,

It would be interesting to have a large number of terms of the expression for $\pi$ as a continued fraction with unit numerators, which starts

$$
3+\frac{1}{7+} \frac{1}{15+} \frac{1}{1+} \frac{1}{292+} \frac{1}{1+} \frac{1}{1+} \frac{1}{1+} \cdots,
$$

For a random irrational number (assuming that 'random' can be defined!) the probability that a denominator of the continued fraction is a given positive integer $r$ is easily shown to be $1 / r(r+1)$. Now some irrational numbers are clearly not random. For example, quadratic irrationals recur, and $e$ has a regular pattern. A sufficiently long expression for $\pi$ would indicate whether $\pi$ is random in this sense.

Of course, a continued fraction has the advantage over a decimal that it is independent of the scale of notation.

Yours etc., E. J. F. Primrose

\title{
To the Editor of the Mathematical Gazette
}

\section{DeAR SIR,}

I would like to reply to one of the points raised by Mr. E. H. Lockwood (Math. Gaz., 1958, 42, 202). As a mathematician, he suggests that we should "teach our pupils to use letters to represent numbers, rather than distances, times or sums of money." As a teacher of chemistry I, along with many other teachers of the physical sciences, among whom I cite, in particular, Professor E. A. Guggenheim, instruct students in what Professor Guggenheim aptly terms the quantity calculus (Journal of Chemical Education, 1958, 35, 606). It appears that the quantity calculus originated in the writings of A. Lodge (Nature, 1888, 38, 281) and J. B. Henderson (Math. Gaz., 1924, 12, 99). In this calculus each letter, like $P$, symbolizes a physical quantity which is represented as the product of a measure (a real number) and an expression (often abbreviated) of the physical units which are being used. There are thus many possible representations of a physical quantity, as in the example $P=1 \mathrm{~atm}=$ $1,013,250$ dynes $\mathrm{cm}^{-2}=1 \cdot 013250$ bar $=1 \cdot 0332275 \mathrm{~kg} . \mathrm{cm}^{-2}=76$ cm mercury $=29.92120$ in. mercury $=14.696006 \mathrm{lb}$. in ${ }^{-2}$. To illustrate I will translate into the language of the quantity calculus the following statement of Mr. Lockwood (loc. cit.). "At $h$ feet above sea level the distance of the horizon is approximately $\sqrt{3 h / 2}$ miles." In terms of the quantity calculus this becomes: "If $h$ and $d$ are the distances above sea level and to the horizon, then $d /$ miles $\simeq$

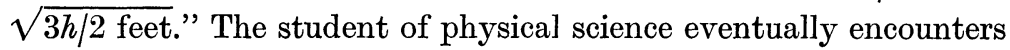


much more involved formulae than this. For instance, in many texts the Sackur-Tetrode entropy formula is written.

$$
\mathrm{s}=\mathbf{R}\left(\frac{5}{2} \ln T+\frac{3}{2} \ln M-\ln P-1 \cdot 164\right),
$$

and a note has to be added stating that $M$ is the gram molecular mass of the gas concerned, $T$ is the absolute temperature in degrees Kelvin $\left({ }^{\circ} \mathbf{K}\right)$ and $P$ is measured in atmospheres. In the quantity calculus equation ( 1 ) and this note are replaced by

$$
\mathrm{s}=\mathbf{R}\left(\frac{5}{2} \ln T /{ }^{\circ} \mathrm{K}+\frac{3}{2} \ln M / \mathrm{g} \cdot \mathrm{mol}^{-1}-\ln P / \mathrm{atm}-1 \cdot 164\right) .
$$

(In (1) and (2) $\mathbf{R}$ is the molar gas constant.) Another advantage of (2) is that it does not commit the error of taking logarithms of physical quantities (see G. N. Copley, Journal of Chemical Education, 1958, 35, 366). Mathematicians insist, I understand, that this should never be done, and although I agree with them and have given, in the reference just cited, one instance of an inconsistency to which this leads, I would like to know of a more general mathematical discussion of the matter.

I hope, therefore, that teachers of mathematics will reconsider the teaching of the quantity calculus in dealing with applied mathematics. Am I right in saying that applied mathematics involves quantity calculus, whereas pure mathematics does not? I am sure that teachers of the physical sciences would welcome criticism of the quantity calculus, since it is most desirable that all teachers should be in agreement upon such an important subject.

Yours etc., G. N. Copley

\section{Dear Sir,}

\section{To the Editor of the Mathematical Gazette}

The Pythagorean musical scale was abandoned in favour of equal temperament because the intervals of the latter, but not of the former, constitute a group. Does any reader know of any other conspicuous contributions made to life outside Mathematics by the elementary theory of groups? (I know about Campanology.)

Yours etc., A. W. FulleR

\section{Wanted}

Mathematical Questions from the Educational Times, 1912 to 1918 inclusive.

Journal of the Indian Mathematical Society, 1909 to 1933 inclusive. Lewent-Conformal Representation.

Hilton-Theory of Groups.

Ganguli-Theory of Plane Curves, Vol. I-3rd ed; Vol. II-2nd ed. McDowell-Exercises in Euclid and Modern Geometry.

Payment in advance. Anyone having any of these to dispose of, may communicate with Clifford Marburger, Denver, Pennsylvania, U.S.A. 